\title{
Second-harmonic generation from complex chiral samples
}

\author{
M. Vanbel ${ }^{1}$, S. Vandendriessche ${ }^{1}$, M. A. van der Veen ${ }^{1,2}$, D. Slavov ${ }^{3}$, P. Heister $^{4}$, R. Paesen ${ }^{5}$, V. K. \\ Valev $^{1}$, M. Ameloot ${ }^{5}$, T. Verbiest ${ }^{1}$ \\ ${ }^{1}$ University of Leuven, Molecular Visualization and Photonics, Celestijnenlaan 200 D, B-3001 \\ Leuven, Belgium \\ ${ }^{2}$ University of Leuven, Centre for Surface Chemistry and Catalysis, Kasteelpark Arenberg 23, B- \\ 3001 Leuven, Belgium \\ ${ }^{3}$ Bulgarian Academy of Sciences, Institute of Electronics, Bulgaria \\ ${ }^{4}$ Department Physikalische Chemie, Technische Universität München , Lichtenbergstrasse 4, 85748 \\ Garching bei München, Germany \\ ${ }^{5}$ Univ Limburg, BIOMED, Univ Hasselt \& Transnatl, B-3590 Diepenbeek, Belgium
}

\begin{abstract}
Second harmonic generation microscopy has recently become an important tool for studying materials. In this article, we use a recently developed analytical method, for second-harmonic generation microscopy, to determine the point group symmetry of micro crystals of enantiomerically pure 1,1'-bi-2-naphtol.
\end{abstract}

Keywords: Second-harmonic generation, microscopy, symmetry, chirality

\section{INTRODUCTION}

Second-harmonic generation ( $\mathrm{SHG}$ ) is a nonlinear optical technique that has been used for many years to study interfaces and surfaces. The reason for its surface sensitivity is that, in the electric dipole approximation, SHG is a second-order nonlinear optical effect that can only be observed in media lacking inversion symmetry. For instance, symmetry is broken by externally applied electric ${ }^{1}$ or magnetic ${ }^{2}$ dc fields, which allows SHG to successfully image ferroelectric ${ }^{3}$ and ferromagnetic ${ }^{4}$ domains. Since inversion symmetry is necessarily broken at an interface, SHG is inherently surface sensitive. SHG is also very sensitive to chirality. For example, very large circular difference effects in SHG have been observed in chiral thin films. ${ }^{5}$ The effect can be considered similar to classical circular dichroism (CD), and therefore it is often referred to as SHG-CD. In addition, a nonlinear optical analogue of optical rotation has also been observed. As a consequence, SHG has been used to study surface chirality and has recently been applied for the study of nano scale systems, biological samples, and mesogenic systems. ${ }^{6,7,9}$

Over the last two decades, SHG has been combined with microscopy, which led to the development of SHG imaging techniques that have become an increasingly popular tool in biological imaging. ${ }^{10,11}$ Additionally, the technique has found applications in the field of materials science, ${ }^{12,13}$ where it has become a successful tool for studying plasmonic materials. ${ }^{14,15}$ An important reason for this success is the fact that SHG microscopy can be used to map plasmonic hotspots in nanostructured materials, ${ }^{16,17,18}$ especially, of chiral geometry. ${ }^{19,20,21}$ More generally, plasmonic hotspots are capable of locally breaking the symmetry of the material and SHG has thus been employed to probe the effects of plasmonic coupling, ${ }^{22,23,24}$ propagation of plasmons along a chain of particles $25,26,27$ and propagating plasmons scattered by metallic nanoparticles. ${ }^{28}$ In the case of plasmonic symmetry breaking, several theoretical models have been proposed.29,30,31,32,33,34 In the case microcrystals, it is especially important to determine the point group symmetry.

It was recently proposed that SHG-microcopy can be used to determine the point group symmetry of noncentrosymmetric samples. ${ }^{35,36}$ The technique is based on a series of simple measurements and does not require a

17th International School on Quantum Electronics: Laser Physics and Applications,

edited by Tanja Dreischuh, Albena Daskalova, Proc. of SPIE Vol. 8770, 87701F

(C) 2013 SPIE - CCC code: 0277-786X/13/\$18 - doi: 10.1117/12.2014745 
complex microscope design. For example, the authors were able to determine the arrangement of dyes located in porous zeolite systems and the symmetry of complex crystalline systems. Here we demonstrate this procedure for microcrystals of 1,1'-bi-2-naphtol (BINOL), and show that even with incomplete experimental data, it is possible to extract valuable information regarding the symmetry of the sample under study.

\section{THEORY}

The method described by van der Veen et al. relies on a series of simple tests using an SHG microscope ${ }^{35,36}$. In order to extract the point group symmetry, typically four different measurements are made. In a first measurement an SHG image is recorded for several linearly polarized input polarizations, while all SHG light, reflected or transmitted is detected. Next several spots in the image are selected and the SHG at those points is plotted against the polarization angle of the input light. This yields a polarization plot in which two observables can be identified, i.e. the number of times the plot reaches zero, and the number of symmetry planes in the plot. The same procedure is then repeated in a second measurement, but in this case the polarized SHG is detected. For measurements 3 and 4 the sample is rotated for fixed input and output polarizations. A schematic of the measurement procedure is shown in Fig. 1. By careful examination of the observables in the four measurements, the point group symmetry can be readily identified. For the case of arbitrarily oriented crystals, i.e. where the crystallographic axes do not coincide with the coordinate system of the sample under study, Table 1 can be used to identify the point group symmetry, based on the observables.
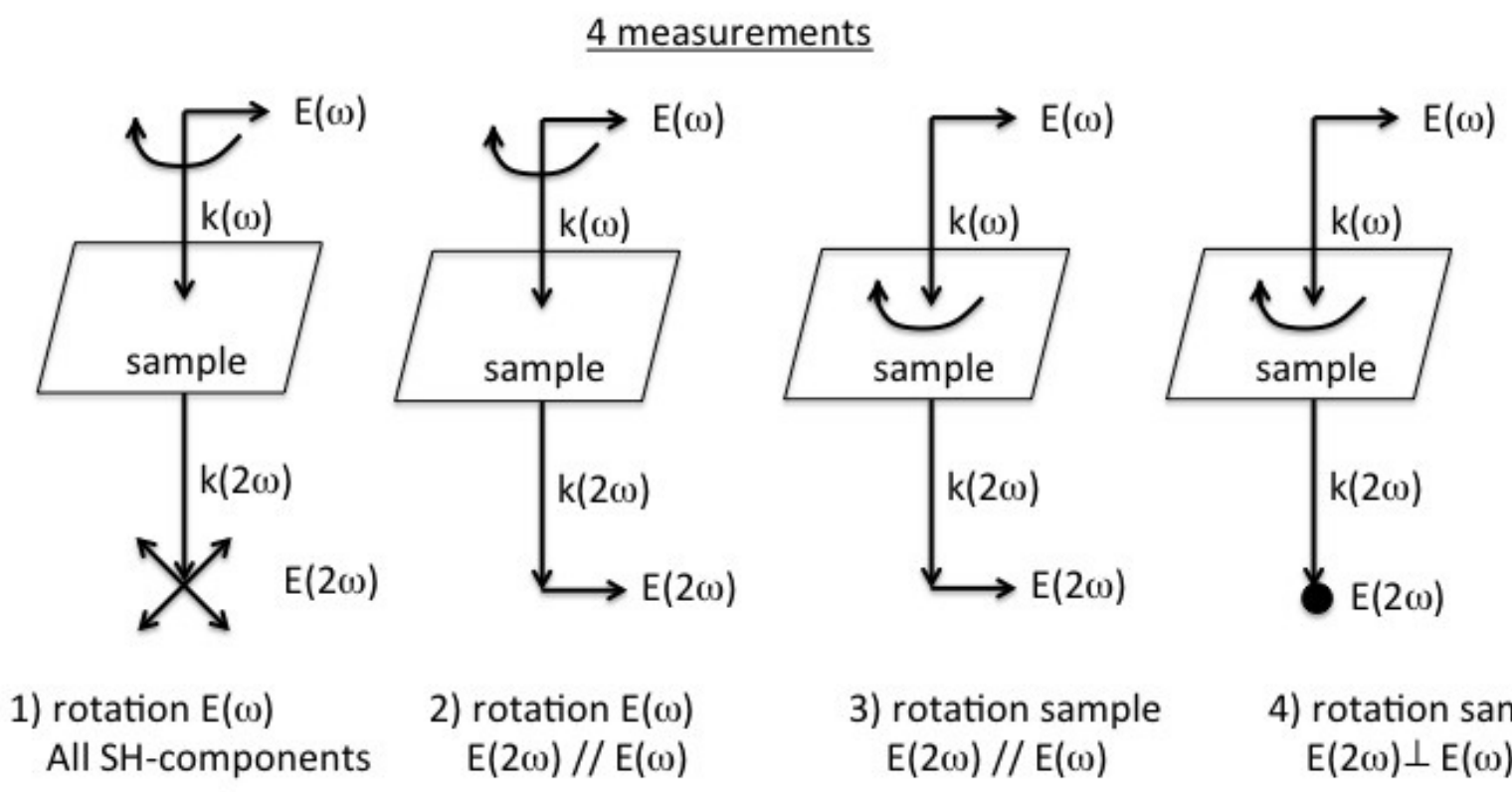
4) rotation sample $E(2 \omega) \perp E(\omega)$

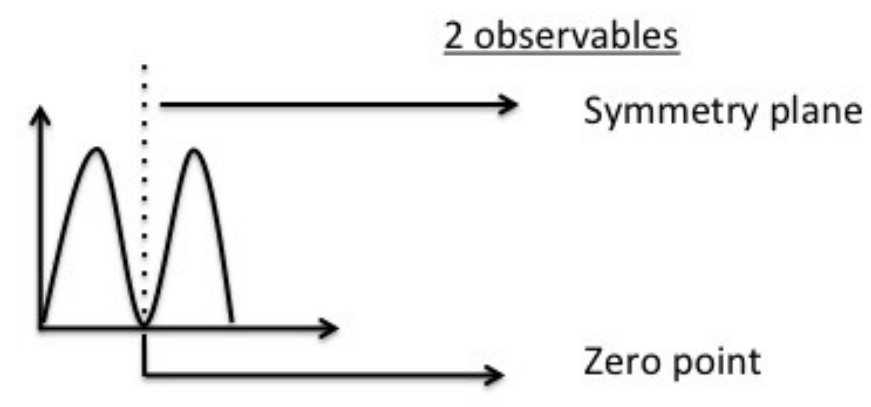

Figure 1: SHG-microscopy and point group determination using 4 measurements and 2 observables 
Table 1: observables for different point group symmetries. $\mathrm{z}=$ zero point, $\mathrm{sp}=$ symmetry plane.

\begin{tabular}{|c|c|c|c|c|}
\hline measurement & 1 & 2 & 3 & 4 \\
\hline $\mathrm{T} / \mathrm{Td}$ & $0 \mathrm{z}, 0 \mathrm{sp}$ & $4 z, 4 s p$ & $6 z, 0 \mathrm{sa}$ & $6 z, 0 \mathrm{sa}$ \\
\hline D6 / D4 /Dœ & $2 z, 4 s p$ & $4 z, 4 s p$ & no SHG & $2 \mathrm{z}, 4 \mathrm{sp}$ \\
\hline $\mathrm{C} 6 \mathrm{v} / \mathrm{C} 4 \mathrm{v} / \mathrm{C} \infty \mathrm{ov}$ & $0 \mathrm{z}, 4 \mathrm{sp}$ & $0 z, 0$ sp & $2 z, 4 s p$ & $2 \mathrm{z}, 4 \mathrm{sp}$ \\
\hline $\mathrm{D} 3 \mathrm{~h}$ & $0 \mathrm{z}, 0 \mathrm{sp}$ & $4 z, 4 s p$ & $6 z, 0 \mathrm{sp}$ & $6 z, 0 \mathrm{sp}$ \\
\hline $\mathrm{C} 6 / \mathrm{C} 4 / \mathrm{C} \infty$ & $0 \mathrm{z}, 0 \mathrm{sp}$ & $0 \mathrm{z}, 0 \mathrm{sp}$ & $2 \mathrm{z}, 4 \mathrm{sp}$ & $0 \mathrm{z}, 0 \mathrm{sa}$ \\
\hline $\mathrm{C} 3 \mathrm{~h}$ & $0 \mathrm{z}, 0 \mathrm{sp}$ & $0 \mathrm{z}, 0 \mathrm{sp}$ & $0 \mathrm{z}, 0 \mathrm{sp}$ & $0 \mathrm{z}, 0 \mathrm{sp}$ \\
\hline D3 & $0 \mathrm{z}, 0 \mathrm{sp}$ & $0 \mathrm{z}, 0 \mathrm{sp}$ & $6 z, 0 \mathrm{sp}$ & $0 \mathrm{z}, 0 \mathrm{sp}$ \\
\hline $\mathrm{C} 3 \mathrm{v}$ & $0 \mathrm{z}, 0 \mathrm{sp}$ & $0 \mathrm{z}, 0 \mathrm{sp}$ & $0 \mathrm{z}, 0 \mathrm{sp}$ & $0 \mathrm{z}, 0 \mathrm{sp}$ \\
\hline $\mathrm{C} 3$ & $0 z, 0 \mathrm{sp}$ & $0 \mathrm{z}, 0 \mathrm{sp}$ & $0 \mathrm{z}, 0 \mathrm{sp}$ & $0 z, 0$ sp \\
\hline D2d & $0 \mathrm{z}, 0 \mathrm{sp}$ & $0 \mathrm{z}, 0 \mathrm{sp}$ & $6 \mathrm{z}, 0 \mathrm{sp}$ & $2 \mathrm{z}, 0 \mathrm{sp}$ \\
\hline S4 / Dœh & $0 \mathrm{z}, 0 \mathrm{sp}$ & $0 \mathrm{z}, 0 \mathrm{sp}$ & $2 \mathrm{z}, 0 \mathrm{sp}$ & $0 \mathrm{z}, 0 \mathrm{sp}$ \\
\hline D2 & $0 \mathrm{z}, 0 \mathrm{sp}$ & $0 \mathrm{z}, 0 \mathrm{sp}$ & $6 z, 0 \mathrm{sp}$ & $0 \mathrm{z}, 0 \mathrm{sp}$ \\
\hline $\mathrm{C} 2 \mathrm{v}$ & $0 \mathrm{z}, 0 \mathrm{sp}$ & $0 \mathrm{z}, 0 \mathrm{sp}$ & $2 \mathrm{z}, 0 \mathrm{sp}$ & $0 \mathrm{z}, 0 \mathrm{sp}$ \\
\hline $\mathrm{C} 2$ & $0 \mathrm{z}, 0 \mathrm{sp}$ & $0 z, 0 \mathrm{sp}$ & $2 z, 0 \mathrm{sp}$ & $0 z, 0 \mathrm{sp}$ \\
\hline Cs & $0 \mathrm{z}, 0 \mathrm{sp}$ & $0 \mathrm{z}, 0 \mathrm{sp}$ & $0 \mathrm{z}, 0 \mathrm{sp}$ & $0 \mathrm{z}, 0 \mathrm{sp}$ \\
\hline $\mathrm{C} 1$ & $0 \mathrm{z}, 0 \mathrm{sp}$ & $0 \mathrm{z}, 0 \mathrm{sp}$ & $0 \mathrm{z}, 0 \mathrm{sp}$ & $0 \mathrm{z}, 0 \mathrm{sp}$ \\
\hline
\end{tabular}

\section{RESULTS AND DISCUSSION}

Here we demonstrate the procedure for a vapor deposited thin film of enantiomerically pure BINOL that was left to crystallize at room temperature. The sample was first investigated by bright field, dark field and polarized microscopy using a commercial Zeiss optical microscope (Axio imager M1). A series of typical images are shown in Fig. 2. It is clear that the sample consists of crystalized regions of star shaped features composed of different needle-like crystals. The polarized images emphasize the anisotropy of the star shaped structures with well-resolved individual crystals. The dark field images on the other hand show the relief on the surface. Especially interesting are the dark field images using a single laser wavelength. Three different diode lasers were used as a light source and could in principle be used to excite fluorescence in the samples, providing additional information on the surface of the structures. 
(a)

Bright field microscopy

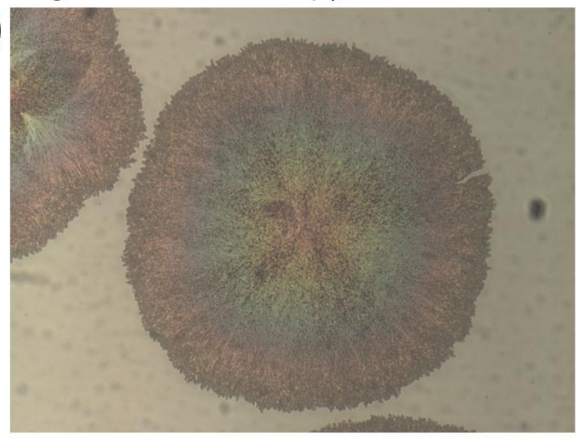

Polarizing microscopy, crossed polarizers

(b)

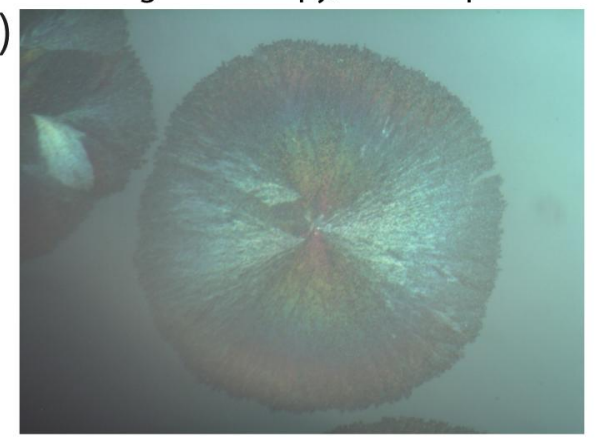

(c)

Polarizing microscopy, analyzer at $+5^{\circ}$

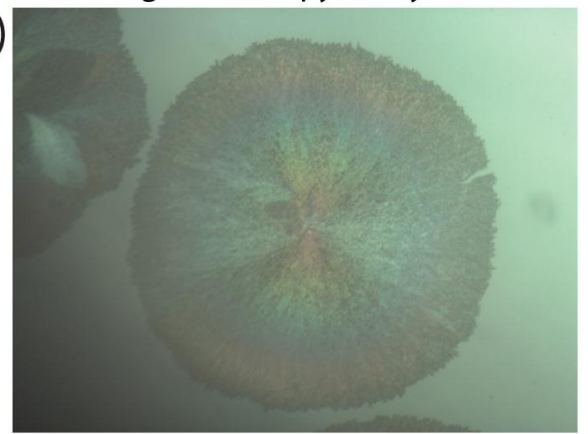

Polarizing microscopy, analyzer at $-5^{\circ}$

(d)

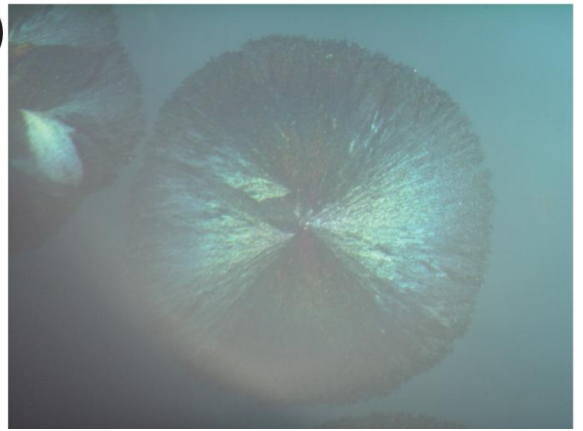

Dark field microscopy with white light

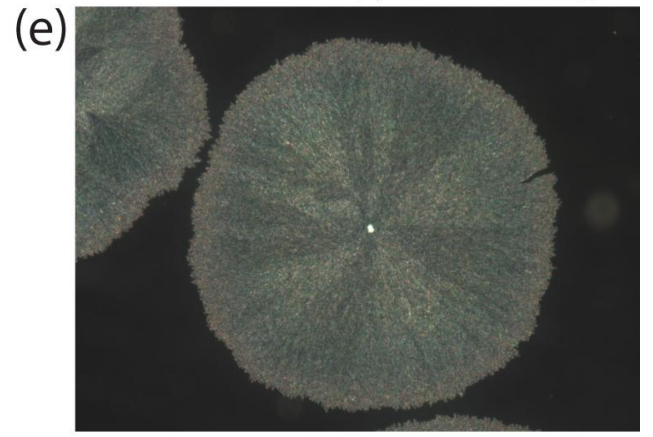

Dark field microscopy at $\lambda=405 \mathrm{~nm}$

(f)

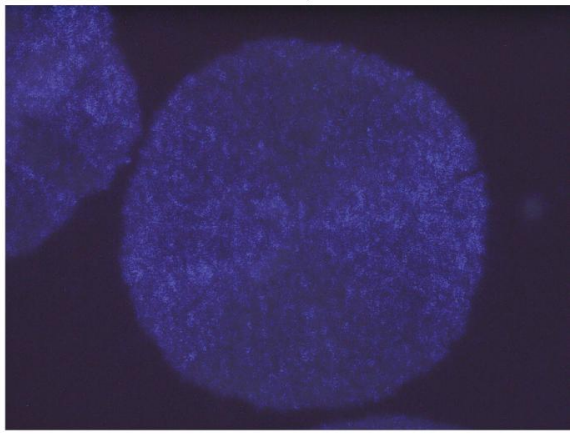

Dark field microscopy at $\lambda=532 \mathrm{~nm}$

(g)

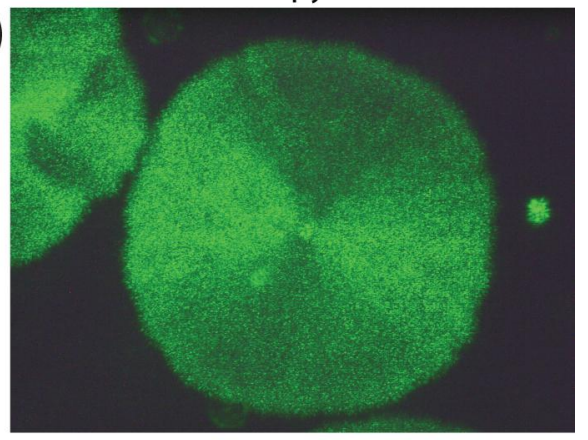

Dark field microscopy at $\lambda=632 \mathrm{~nm}$

(h)

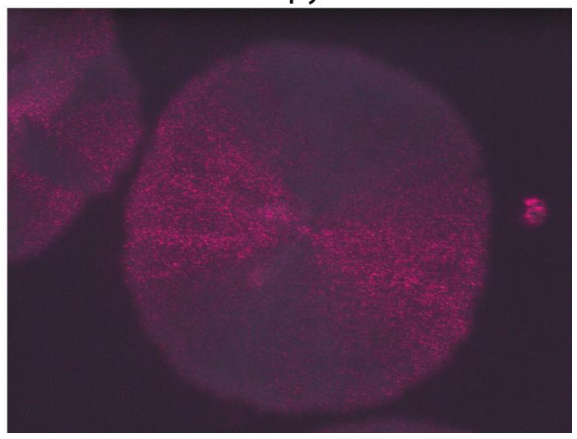

Figure 2: optical microscopy of crystallized samples of BINOL. The actual size of the images is 700x700um

SHG images were taken with a commercial Zeiss LSM 510 confocal microscope and an example is shown in Fig. 3. Noteworthy is the drastically improved resolution as compared to the optical microscopy images. Furthermore, the 
anisotropy is more strongly emphasized as compared to the optical microscopy images, illustrating the strength of SHG microscopy. Next we performed two types of measurements in which several images with different input polarization were taken, with and without analyzer, and the SHG intensity of a selected crystal was plotted $v s$. the angle of the input polarization. This is shown in Figure 4. Because of experimental limitations we could not perform measurements in which the sample was rotated. However, due to the star like arrangement of the needle like crystals, we could analyze the SHG from different needle like crystals as a function of the angle they make with respect to the input polarization. Hence, if we assume that all crystals are identical, this is in principle equivalent to a rotation of the sample. The corresponding plots are also shown in Fig 4.

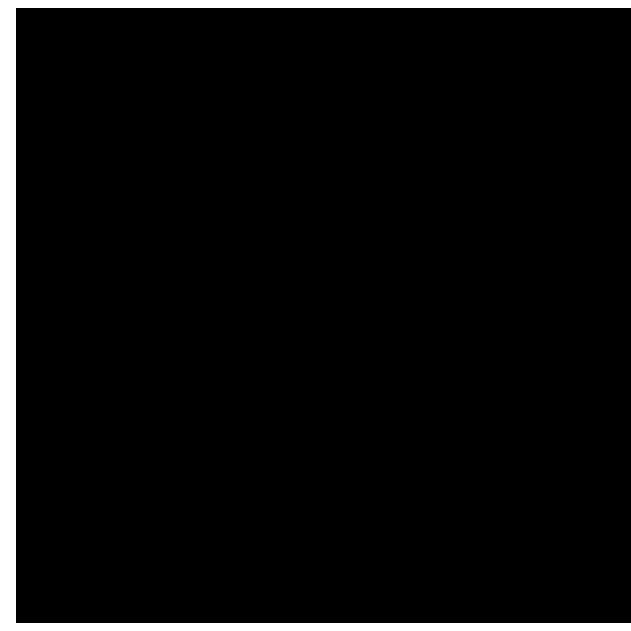

Figure 3: SHG image of crystallized binol

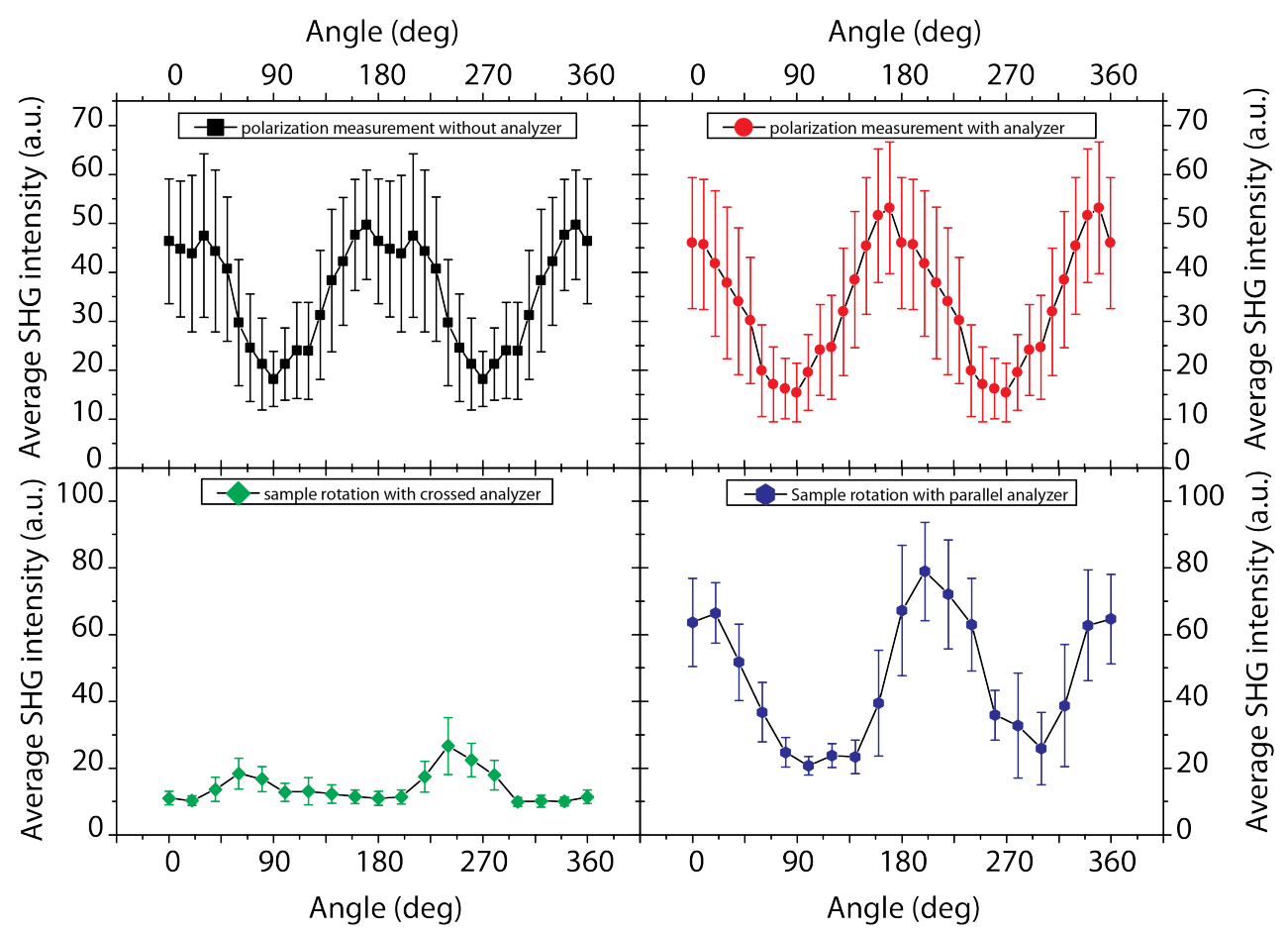

Figure 4: the top images are polarization plots corresponding to measurement 1 and 2 . The bottom images were extracted from measurements 1 and 2 with each data point corresponding to a crystal with a different orientation with respect to the input polarization, effectively simulating sample rotation. 
The next step in our analysis consists of identifying the number of times each plot goes to zero and the number of symmetry planes that can be drawn through the plot. However, due to the inherent uncertainty on each data point it is difficult to establish the presence of symmetry planes in the polarization graphs. Nevertheless, none of the polarization plots go to zero, thus limiting the number of possible symmetry point groups. From Table 1 we find that the only possible point groups are $\mathrm{C}_{3 \mathrm{~h}}, \mathrm{C}_{3 \mathrm{v}}, \mathrm{C}_{3}, \mathrm{C}_{\mathrm{S}}$ and $\mathrm{C}_{1}$. Given the fact that the sample under study is enantiomerically pure BINOL, this leaves us with either a $\mathrm{C}_{3}$ or $\mathrm{C}_{1}$ symmetry. This is in perfect agreement with $\mathrm{x}$-ray diffraction data that indicate a $\mathrm{C}_{3}$ symmetry. ${ }^{37}$

\section{CONCLUSION}

We have demonstrated the potential of a recently developed technique, based on SHG-microscopy- to determine the point group symmetry of very complex samples. Furthermore, we demonstrated that even with incomplete data, valuable information concerning the symmetry of the samples can be extracted.

\section{ACKNOWLEDGEMENTS}

We acknowledge financial support from the Fund for scientific research Flanders (FWO-V), the FWO-BAS exchange program and the K.U. Leuven (GOA). V.K.V., M.A. vdV. and S.V. are grateful for the support from the FWOVlaanderen. P.H. acknowledges support from the TUM graduate school. M.A. obtained support from the tUL Impuls plan II.

\section{REFERENCES}

[1] Lee, C. H., Chang, R. K. and Bloembergen, N., "Nonlinear electroreflectance in silicon and silver," Phys. Rev. Lett. 18(5), 167170 (1967).

[2] Kirilyuk, A. and Rasing, Th., "Magnetization-induced-second-harmonic generation from surfaces and interfaces,” J. Opt. Soc. Am. B 22(1), 148-167 (2005).

[3] Sheng, Y., Best, A., Butt, H.-J., Krolikowski, W., Arie, A. and Koynov, K., "Three-dimensional ferroelectric domain visualization by Cerenkov-type second harmonic generation," Opt. Express 18(16), 16539-16545 (2010).

[4] Pavlov, V. V., Ferré, J., Meyer, P., Tessier, G., Georges, P., Brun, A., Beauvillain, P. and Mathet, V., "Linear and non-linear magneto-optical studies of Pt/Co/Pt thin films," J. Phys. Condens. Matter 13(44), 9867-9878 (2001).

[5] Verbiest, T., Clays, K., Rodriguez, V, Second-order Nonlinear Optical Characterization Techniques: An Introduction, CRC Press, Boca Raton (2009)

[6] Valev, V. K., Silhanek, A. V., Verellen, N., Gillijns, W., Vandenbosch, G.A.E., Aktsipetrov, O. A., Moshchalkov, V. V. and Verbiest, T., "Asymmetric Second Harmonic Generation from Chiral G-Shaped Gold Nanostructures," Phys. Rev. Lett., 104, 127401

(2010).

[7] Salafsky, J.S., "Second-harmonic generation for studying structural motion of biological molecules in real time and space," Phys. Chem. Chem. Phys. 9, 5704-5711 (2007).

[8] Van Cleuvenbergen, S., Hennrich, G., Willot, P., Koeckelberghs, G., Clays, K., Verbiest, T. and van der Veen, M.A., "All Optical Determination of Microscopic and Macroscopic Structure of Chiral, Polar Microcrystals from Achiral, Nonpolar Molecules," J. Phys. Chem. C, 116, 12219-12225 (2012)

[9] Araoka, F., Ha, N.Y., Kinoshita, Y., Park, B., Wu, J.W. and Takezoe, H., "Twist-grain-boundary structure in the B4 phase of a bent-core molecular system identified by SHG circular dichroism measurement," Phys. Rev. Lett. 94, 137801 (2005).

[10] Zoumi, A., Yeh, A., Tromberg, B.J., "Imaging cells and extracellular matrix in vivo by using second-harmonic generation and two-photon excited fluorescence," Proc. Nat. Acad. Sci. 99, 11014-11019 (2002).

[11] Campagnola, P.J., Loew, L.M., "Second-harmonic imaging microscopy for visualizing biomolecular arrays in cells, tissues and organisms," Nature Biotech. 21, 1356-1360 (2003)

[12] Zayats, A.V., Smolyaninov, I.I. and Davis, C.C., "Observation of localized plasmonic excitations in thin metal films with nearfield second-harmonic microscopy," Opt. Commun. 169, 93-96 (1999).

[13] Anceau, C., Brasselet, S., Zyss, J. and Gadenne, P., "Local second-harmonic generation enhancement on gold nanostructures probed by two-photon microscopy," Opt. Lett. 28, 713-715 (2003).

[14] Butet, J., Duboisset, J., Bachelier, G., Russier-Antoine, I., Benichou, E., Jonin, C. and Brevet, P.-F. “Optical Second Harmonic Generation of Single Metallic Nanoparticles Embedded in a Homogeneous Medium," Nano Lett. 10, 1717-1721 (2010). 
[15] Butet, J., Bachelier, G., Duboisset, J., Bertorelle, F., Russier-Antoine, I., Jonin, C., Benichou, E. and Brevet, P.-F., "Threedimensional mapping of single gold nanoparticles embedded in a homogeneous transparent matrix using optical second-harmonic generation," Opt. Express 18, 22314-22323 (2010).

[16] Valev, V. K., Silhanek, A. V., De Clercq, B., Gillijns, W., Jeyaram, Y., Zheng, X., Volskiy, V., Aktsipetrov, O. A., Vandenbosch, G. A. E., Ameloot, M., Moshchalkov, V. V. and Verbiest, T., "U-shaped switches for optical information processing at the nanoscale," Small 7, 2573-2576 (2011).

[17] Valev, V. K., Denkova, D., Zheng, X., Kuznetsov, A. I., Reinhardt, C., Chichkov, B. N., Tsutsumanova, G., Osley, E.J., Petkov, V., De Clercq, B., Silhanek, A. V., Jeyaram, Y., Volskiy, V., Warburton, P. A., Vandenbosch, G. A. E., Russev, S., Aktsipetrov, O.

A., Ameloot, M., Moshchalkov, V. V. and Verbiest, T., "Plasmon-enhanced sub-wavelength laser ablation: plasmonic nanojets," Adv. Mater. 24, OP29-OP35 (2012).

[18] Valev, V. K., De Clercq, B., Biris, C. G., Zheng, X., Vandendriessche, S., Hojeij, M., Denkova, D., Jeyaram, Y., Panoiu, N. C., Ekinci, Y., Silhanek, A. V., Volskiy, V., Vandenbosch, G. A. E., Ameloot, M., Moshchalkov, V. V. and Verbiest, T.,"Distributing the optical near field for efficient field-enhancements in nanostructures," Adv. Mater. 24, OP208-OP215 (2012).

[19] Valev, V. K., De Clercq, B., Zheng, X., Denkova, D., Osley, E. J., Vandendriessche, S., Silhanek, A. V., Volskiy, V., Warburton, P. A., Vandenbosch, G. A. E., Ameloot, M., Moshchalkov, V. V. and Verbiest, T., "The role of chiral local field enhancements below the resolution limit of Second Harmonic Generation microscopy," Opt. Express 20, 256-264 (2012).

[20] Huttunen, M.J., Bautista, G., Decker, M., Linden, S., Wegener, M. and Kauranen, M., "Nonlinear chiral imaging of subwavelength-sized twisted-cross gold nanodimers," Opt. Mat. Express 1, 46-56 (2011).

[21] Valev, V.K. "Characterization of Nanostructured Plasmonic Surfaces with Second Harmonic Generation [Invited Feature Article]," Langmuir (2012), doi: 10.1021/la302485c.

[22] Benedetti, A., Centini, M., Sibilia, C. and Bertolotti, M., "Engineering the second harmonic generation pattern from coupled gold nanowires," J. Opt. Soc. Am. B 27(3), 408-416 (2010).

[23] Centini, M., Benedetti, A., Sibilia, C. and Bertolotti, M., "Coupled 2D Ag nano-resonator chains for enhanced and spatially tailored second harmonic generation," Opt. Express 19, 8218-8232 (2011).

[24] Belardini, A., Larciprete, M. C., Centini, M., Fazio, E., Sibilia, C., Bertolotti, M., Toma, A., Chiappe, D. and Buatier de Mongeot, F., "Tailored second harmonic generation from self-organized metal nano-wires arrays," Opt. Express 17(5), 3603-3609 (2009).

[25] Biris, C.G. and Panoiu, N. C., "Excitation of dark plasmonic cavity modes via nonlinearly induced dipoles: applications to nearinfrared plasmonic sensing," Nanotechnology 22, 235502 (2011).

[26] Biris, C. G. and Panoiu, N. C, "Excitation of linear and nonlinear cavity modes upon interaction of femtosecond pulses with arrays of metallic nanowires," Appl Phys A 103, 863-867 (2011).

[27] Awada, Ch., Kessi, F. , Jonin, Ch., Adam, P. M., Kostcheev, S., Bachelot, R., Royer, P., Russier-Antoine, I., Benichou, E., Bachelier, G. and Brevet, P. F., "On- and off-axis second harmonic generation from an array of gold metallic nanocylinders," J. Appl. Phys. 110, 023109 (2011).

[28] Cao, L., Panoiu, N. C. and Osgood, R. M., "Surface second-harmonic generation from surface plasmon waves scattered by metallic nanostructures," Phys. Rev. B 75, 205401 (2007).

[29] Cao, L., Panoiu, N. C., Bhat, R.D.R. and Osgood, R.M., "Surface second-harmonic generation from scattering of surface plasmon polaritons from radially symmetric nanostructures," Phys. Rev. B 79, 235416 (2009).

[30] Biris, C.G. and Panoiu, N.C., "Second harmonic generation in metamaterials based on homogeneous centrosymmetric nanowires," Phys. Rev. B 81, 195102 (2010).

[31] Dadap, J. I., Shan, J., Eisenthal, K. B. and Heinz, T. F., "Second-harmonic Rayleigh scattering from a sphere of centrosymmetric material," Phys. Rev. Lett. 83(20), 4045-4048 (1999).

[32] Zeng, Y., Hoyer, W., Liu, J., Koch, S. W. and Moloney, J. V., "Classical theory for second-harmonic generation from metallic nanoparticles," Phys. Rev. B 79(23), 235109 (2009).

[33] Schaich, W. L., "Second harmonic generation by periodically-structured metal surfaces," Phys. Rev. B 78(19), 195416 (2008).

[34] Mäkitalo, J., Suuriniemi, S. and Kauranen, M., "Boundary element method for surface nonlinear optics of nanoparticles," Opt. Express 19, 23386 (2011).

[35] M.A. van der Veen, F. Vermoortele, D. E. De Vos, T. Verbiest, Anal Chem, 84, 6378-6385 (2012).

[36] M.A. van der Veen, F. Vermoortele, D. E. De Vos, T. Verbiest, Anal Chem, 84, 6386-6390 (2012).

[37] Lee, T.; Peng, J. F. Cryst. Growth Des. 2010, 10, 3547-3554. 